\title{
A review of biomedical and health informatics education: A workforce training framework
}

\author{
Saif Khairat*1, Ryan Sandefer ${ }^{2,3}$, David Marc ${ }^{2,3}$, Lee Pyles ${ }^{4}$ \\ ${ }^{1}$ Carolina Health Informatics Program, University of North Carolina-Chapel Hill, Chapel Hill, NC, United States \\ ${ }^{2}$ 2Institute for Health Informatics, University of Minnesota, Minneapolis, MN, United States \\ ${ }^{3}$ The College of St. Scholastica, Duluth, MN, United States \\ ${ }^{4}$ Department of Pediatrics, West Virginia University, Morgantown, WV, United States
}

Received: April 24, 2016

DOI: $10.5430 /$ jha.v $5 \mathrm{n} 5 \mathrm{p} 10$
Accepted: June 24, $2016 \quad$ Online Published: July 4, 2016

URL: http://dx.doi.org/10.5430/jha.v5n5p10

\begin{abstract}
Objective: The purpose of this paper is to review the current state of health information technology (HIT) training programs and identify limitations in workforce expectations and student/trainee level of preparedness. A framework is proposed to build a more effective training program, differentiate HIT and health informatics, and emphasize the critical role of interprofessional collaboration for informatics-related curriculum. We define interprofessionalism as the multi-sector collaborations among academia, industry (Health Care Organizations), and vendors to produce competent informaticians.

Methods: Critical review of published HIT and health informatics curricular competencies was conducted, including those published by the Office of the National Coordinator (ONC) for HIT, the American Medical Informatics Association (AMIA), the International Medical Informatics Association (IMIA), and the Council on Accreditation for Health Informatics and Information Management. A review of literature related to HIT and health informatics education and training was also completed.

Results: The paper presents a framework for promoting health informatics training with an interprofessional foundation. The core components of the curricular competencies include understanding the healthcare system, biomedical data, computer programming, data analytics, usability, and technology infrastructure. To effectively deliver the content, programs require collaboration between academic institutions, healthcare organizations, and industry vendors.

Conclusions: HIT and health informatics-related training programs, in their current form, are not meeting industry needs. The proposed framework addresses the current limitations by providing unique pathways for content delivery by promoting interprofessional collaboration and partnerships between academia and industry.
\end{abstract}

Key Words: Health, Medical, Informatics, Workforce, Training

\section{INTRODUCTION}

The Health Information Technology (HIT) for Economic and Clinical Health (HITECH) Act, enacted as a component of the American Recovery and Reinvestment Act of 2009, had a sole purpose to promote the adoption and meaningful use of HIT. The HITECH Act was designed in a manner with the understanding that the current workforce was inadequate for meeting the needs of an electronic healthcare system. The anticipated growth in the use of Electronic Health Records (EHR) systems is creating a demand for health IT (HIT) workers who are prepared to provide installation services, workflow redesign, and the support of activities such as qual-

*Correspondence: Saif Khairat, PhD; Email: saif@unc.edu; Address: Carolina Health Informatics Program, University of North Carolina-Chapel Hill, Chapel Hill, NC, United States. 
ity reporting and other key aspects comprising the meaningful use of EHRs. Thus, the supply of qualified health informatics professionals is a factor that can limit the rate at which certified EHR technology can be adopted, and may be one of the greatest barriers to comprehensive adoption and meaningful use of HIT. This paper refers to education as reinforcing knowledge in which that a student may or may not have as foundation; while training is defined as providing hands-on experiences that are applicable to real-life scenarios.

At the time of the HITECH Act's passage, there was a projected shortfall of 50,000 HIT professionals who could work with providers in the clinic and at the bedside to ease the transition into the electronic world. ${ }^{[1]}$ Since the passage of the HITECH Act, the number of online HIT job postings has nearly tripled. ${ }^{[2]}$ To meet the workforce demands the HITECH Act was clearly going to create the Office of the National Coordinator (ONC) designed and included workforce development training programs within the legislation. Of the HITECH Act's \$26 billion dollars in programs, \$120 million was designated for workforce programs.

The ONC defined 12 professional roles to be targeted across 4 different development training programs each differing in timelines and curriculum. The $\mathrm{ONC}$ defined the 4 programs as such: ${ }^{[3]}$

Community College Consortia (CCC): Five regional groups of 82 member community colleges were represented across the United States. These colleges received funding to rapidly create HIT training programs or expand existing programs which could be completed by incoming students in six months or less. The programs were designed for students with a previous background in IT or health care and meant to fulfil the following professional roles:

- Practice workflow and information management redesign specialist;

- Clinician/practitioner consultants;

- Implementation support specialists;

- Implementation managers;

- Technical/software support, and trainers.

The goal was to train 10,500 new HIT specialists by 2012 .

\section{Informatics and HIT workforces - interprofessional competencies}

There is a clear and marked difference between HIT Professionals and Informaticians, yet it is clear that the ONC workforce roles outlined above are intended to address a shortage of informatics-related competencies. The developers of the $\mathrm{CCC}$ and university-based training (UBT) curriculum cate- gorized the 12 roles into three "informatics" workforce categories: (1) mobile adoption support roles; (2) permanent staff of healthcare delivery support roles; and (3) healthcare and public health informaticians. ${ }^{[4]}$ The main difference between HIT and Informatics workforces is the delivery and execution of technological solutions to a given problem compared to assessing and improving the effectiveness of healthcare services and outcomes through building knowledge bases. Despite the differences between HIT and Informatics professionals, both professionals have at their core the need to be interprofessionally competent. According to a white paper drafted by the American Medical Informatics Board, the ability to "work collaboratively" and to "team effectively with partners within and across disciplines" is a core competency of informatics professionals. ${ }^{[5]}$ Similarly, the Institute of Medicine, the World Health Organization, and the Interprofessional Education Collaborative have noted "teams and teamwork" as a core competency for all healthcare professionals. ${ }^{[6]}$

HIT is the utilization of an array of technologies to store, share, and analyze information. ${ }^{[7]}$ According to the American Medical Informatics Association (AMIA), biomedical informatics is the "interdisciplinary field that studies and pursues the effective uses of biomedical data, information, and knowledge for scientific inquiry, problem solving, and decision making, driven by efforts to improve human health". ${ }^{[5]}$ While both definitions contain overlapping concepts, the differences are substantial. HIT's primary focus is to design and develop technological advances that fit the demands of the healthcare industry therefore; the training of HIT professionals is mostly geared towards necessary technical and computational concepts and methods, while exposure to the clinical point of view of the problem may lack. Informatics is the science of improving health care services and outcomes through better understanding of clinical data and incorporation of various computational methods. Informaticians are expected to have the ability to decipher large clinical datasets combined with the ability to identify the correct computational methods to tackle a given healthcare problem. Friedman clearly illustrates the difference between HIT and informatics and he describes health informatics as a "cross-training" between basic information sciences (e.g. organizational science) and specific domains of application (e.g. public health). ${ }^{[8]} \mathrm{He}$ argues that "cross-training spawns unique forms of creative potential and problem-solving capability that grow out of the connections the mind establishes when different areas of knowledge are invoked simultaneously. Cross-training also enables communication with both the basic scientists and the full-time professionals, making it possible for the cross-trained person to promote important 
modes of collaboration". ${ }^{[8]}$

Vendors and health organizations differentiate between HIT professionals and Health Informatics. The general consensus is that HIT is more programming and computational based, while Informatician is inclined towards individuals with a firm understanding of clinical workflows and healthcare management. Thus, exact replication of HIT training models to the Informatics arena will not yield a trained workforce that meets the needs of the community. In an interprofessional field like Health Informatics, there needs to be a tailored training model rather than one-size fits all model. For these reasons, this paper proposes a vision towards further development of a hybrid educational framework that would take in individuals with undergraduate degrees in areas such as health sciences or IT; and train them to become Informaticians by the time of completion.

\section{ANALYTICAL REVIEW}

As the aforementioned reports and studies show that current gaps in HIT workforce development are multifaceted. This study explored current HIT educational and training programs, evaluated program outcomes, and highlight that successful programs require collaborative efforts to equip trainees with informatics competencies. An area of weakness in healthcare is the modest levels of engagement between universities and colleges, hospital and medical centers, and private vendors.

\subsection{Program of assistance for UBT}

The UBT was meant to fill gaps in HIT roles where university-level training is required. Nine different colleges/universities were selected to rapidly establish new or expanding training programs to quickly provide training to students so that they graduate well prepared for their chosen HIT professional roles. The programs were to be completed in one year or less. The UBT programs were meant to fulfil the following professional roles:

- Clinician/public health leader;

- Health information management and exchange specialist;

- Health information privacy and security specialist;

- Research and development scientist;

- Programmers and software engineer;

- HIT sub-specialist.

\subsection{Curriculum development centers}

The purpose of these centers was to provide funding to institutions of higher education to support HIT curriculum development. Five institutions of higher education were selected for the curriculum development. The curriculum was meant to enhance workforce training programs primarily at the community college level.

Competency Examinations: Six examinations were developed to assess basic competencies in one of six HIT workforce roles. The exams aligned with the roles and training provided by the CCC. The exams consisted of 125 multiplechoice questions and were to be completed in a 3-hour timeframe. The goals of the exam were to validate individual and organizational competencies, lower cost of staff development, and garner organizational credibility.

Four years into the workforce development programs yet there is still a need to provide more adequate training for HIT professionals. The workforce is in need of a greater quantity of HIT professionals but also requires improvements in the quality of the individuals being trained. Unfortunately, some training programs focus more on breadth, while others focus on depth; there is a need for a balanced program design that provides adequate breadth and depth. ${ }^{[9]}$ As a result, HIT professionals are often left unprepared for the workforce demands. The purpose of this paper is to review the current state of HIT training programs and identify limitations in workforce expectations and student/trainee level of preparedness. This paper also provides a framework to build a more effective training program.

\subsection{Current state of HIT training programs}

The National Opinion Research Center (NORC) at the University of Chicago was tasked with annually evaluating the efficacy of the ONC training programs in totality, but also individually. The most recent report was released in September 2013. ${ }^{[10]}$ The 2013 report focused on describing the implementation efforts of the training programs using qualitative data from surveys, focus groups, and interviews with students, program administrators and staff, faculty members, and employers. They found that 20,238 students successfully completed or were currently enrolled in the CCC program. Moreover, the report illustrated the challenges of educating the HIT workforce through the program's architecture, $38 \%$ of all students enrolled in the CCC program either withdrew or failed the program. Another shortcoming of the CCC programs was that they did not appear to be closing the urban-rural divide in terms of HIT workforce. According to the ONC HIT Dashboard, approximately $94 \%$ of all program trainees resided in urban areas (metropolitan or micropolitan areas), 5\% lived in rural areas, and $1 \%$ were unknown. ${ }^{[1]}$ Those with prior HIT experience accounted for $24 \%$ of the students, healthcare background accounted for $38 \%$ of the students, and $21 \%$ of the students had an IT background without any healthcare experience. The rationale behind significant student retention is not well documented 
in the literature; contributing factors may include financial aid, change in geographic location, or change in educational preferences. Both the CCC and UBT were found to have diverse curricula fully online, partially online, or in-class while offering training for roles they decided were within the scope of the curriculum. It was noted that one of the consortia offered two different tracks depending on the students' background: an engineering track for students with a background in IT and a consulting track for students with a healthcare background. ${ }^{[3]}$ Although the CCC and UBT were similar in many regards, the UBT programs were typically designed to award a master's degree and in some cases additional graduate certifications. Finally, some of the CCC offered employer outreach with internships and developing relationships with professional organizations. The UBT provided employer outreach with job fairs and relationships with professional organizations.

Regarding the curriculum development centers, the curriculum materials were typically created with PowerPoint slides with voice-over narration and recordings, class activities and homework assignments, self-assessments, and links to supplemental readings and other resources. The Centers created "blueprints" for guiding the CCC and some colleges and universities in determining which training materials would be most appropriate for each training role. The Centers had diverse methods for reviewing the materials including advisory boards, student reviewing, gathering input from other Centers, local employers, universities, and the ONC. The feedback they obtained through the reviewing processes helped with revisions of the materials for subsequent releases.

The HIT competency exams were delivered to 3,771 individuals (less than $30 \%$ of all training program graduates) across the various roles with an additional 293 scheduled. The majority of the test takers had a bachelor's degree, nearly half had an IT background, and the nearly half had a health care background. Interestingly, $19 \%$ of the test takers were unemployed. Some of the reasons for taking the exam included: to test themselves against a national standard; gain validation for what they felt they had learned on the job; test gaps in their personal knowledge base; and support career development/advancement.

It appears that those individuals who either have not found work or were not already employed before entering the program are more likely to sit for this examination. According to the NORC evaluation, the percentage of CCC students who were employed pre- and post-program has increased across all three cohorts of the program (see Figure 1).

While the employment status of program participants improved, the impact of the program on the intended goal of addressing HIT workforce shortages is still in question. According to the NORC evaluation, only between 30\%-40\% of those participants who are employed are working in HIT. Of those working in HIT, $60 \%$ are working for the same employer when they started the program and only $21 \%-25 \%$ of those had a change in role or title. Moreover, the majority of respondents listed their responsibility as simply using an EHR. In other words, the number of program participants who are making major shifts in employment in this sector is marginal. Lastly, only $19 \%-34 \%$ of program graduates report being very satisfied with the program. When asked about potential program improvements, students reported a desire for "additional opportunities for hands-on experience, including internship opportunities". ${ }^{[12]}$

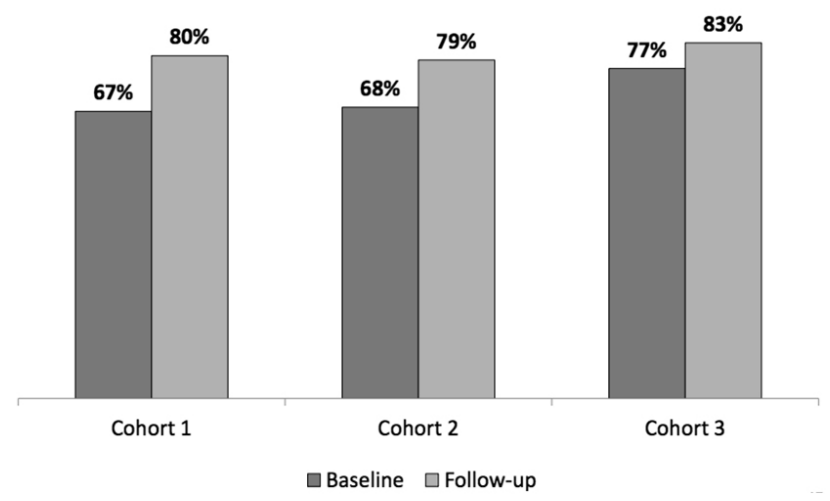

Figure 1. Employment Pre- and Post-CCC Program Participation (HealthIT.gov, 2013b) ${ }^{[11]}$

A small survey of 255 Health Information Exchange (HIE) organizations' hiring practices conducted by the eHealth Initiative in 2012 demonstrates the concern regarding industry acceptance of ONC workforce graduates. According to the study's results, $28 \%$ of HIEs specifically responded that they did not have plans to hire ONC program graduates to fill staff shortages. ${ }^{[13]}$ While this study is focused on HIEs specifically and therefore does not reflect the entirety of the HIT industry, nor does the study differentiate between CCC graduates and UBT graduates, it does indicate a need for further study of the impact and marketability of ONC graduates.

In critically evaluating NORCs efforts in their current evaluation of the ONC training programs, there was very limited information that detailed the trainee's preparedness for the workforce. NORC evaluators did not present any data related to whether employer expectations were met with regard to trainee competencies or the acceptability of the ONC's defined roles. Based upon the information presented in the NORC report, it is difficult to gauge the overall impact of the ONC workforce training program to date. 
Even though the NORC Evaluation did not present favourable findings regarding the impact of the workforce training programs on the industry, perhaps the search for that data is a red herring. The design of the HITECH ONC grant programs to support the Center for Medicare and Medicaid (CMS) EHR Incentive Program (aka "meaningful use"), as illustrated by David Blumenthal in Figure 2 below, was intended to promote connections between and among programs. For example, CCC graduates theoretically were intended to staff Regional Extension Centers, who in turn were intended to support eligible professionals and hospitals use of Certified EHR systems to attain incentive payments.
Figure 2. The design of the HITECH ONC grant programs to support the CMS EHR Incentive Program $^{[1]}$

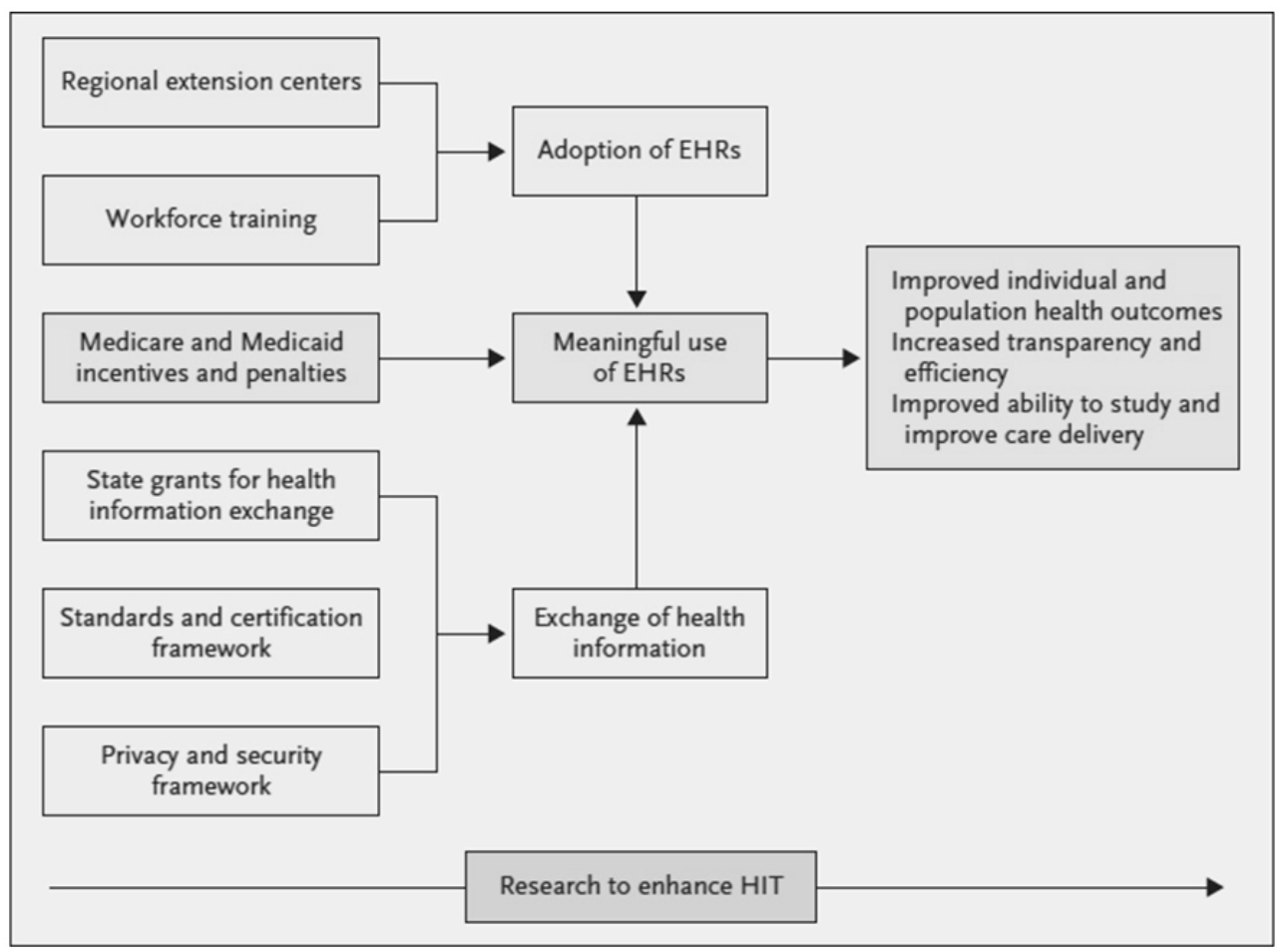

This framework, overall, has not come to fruition. The timelines required for meeting Stage 1 of meaningful use required that vendors be certified, Regional Extension Centers staffed (largely), and healthcare organizations implemented well before the workforce programs had graduated their first cohort of students. Therefore, it could be argued that the ONC's workforce development program was not successful in program design, as the Regional Extension Centers and workforce training programs did not have adequate time to assist the proper adoption of HIT. The overall impact of this design flaw is unknown however, only $1 \%$ of employed graduates of the CCC program are working for Regional Extension Centers. ${ }^{[12]}$ Therefore, this paper proposes a new framework should be developed in order to develop a competent, well-prepared workforce.

Despite the existence of educational programs that train future workforce on informatics competencies, there is still a need for an educational bipartisan framework that better prepares future informatics workforce, and a comprehensive roadmap needs to incorporate informatics awareness into the various disciplines. The preparation of future informati- cians relies heavily on two major axes: (1) education, and (2) training. Currently, health informatics programs recruit students and faculty from multiple professional backgrounds (nursing, health services research, medicine, pharmacy, IT, dentistry, etc.) and utilize curricula across educational disciplines (medicine, public health, computer science, cognitive science, statistics, etc.). While this current model exposes students to multiple disciplines by its nature, a more focused and intentional effort needs to be placed on providing an interprofessional education, including constructing curricula around formal educational programming within academic institutions, technology vendors, and healthcare organizations. As shown in Figure 2, the aforementioned entities each hold a different role in the training process: educational institutions are responsible to educate future informaticians, vendors provide the software products and support, and healthcare organizations provide de-identified datasets for teaching and training purposes and provide internship opportunities for applied informatics work.

Educational institutions have a role in determining the needs and expectations when training informatics workforce 
through discussions with involved clinical and informatics schools. To provide adequate informatics education, an institution must provide an interprofessional curriculum, offer access to EHR systems, and provide access to EHR trained instructors that can accommodate students with various backgrounds. The aim of an interprofessional curriculum is to teach the fundamental concepts of informatics, the functionalities of EHR systems, and the application of EHRrelated technologies such as Clinical Decision Support System (CDSS) or E-Prescribing. Patel and colleagues explained how educational programs that incorporated EHRs into the curriculum have a positive impact on how trainees organize medical information and subsequent reasoning. ${ }^{[14]}$ Thus, the use of technology in health informatics education can impact performance and job preparedness.

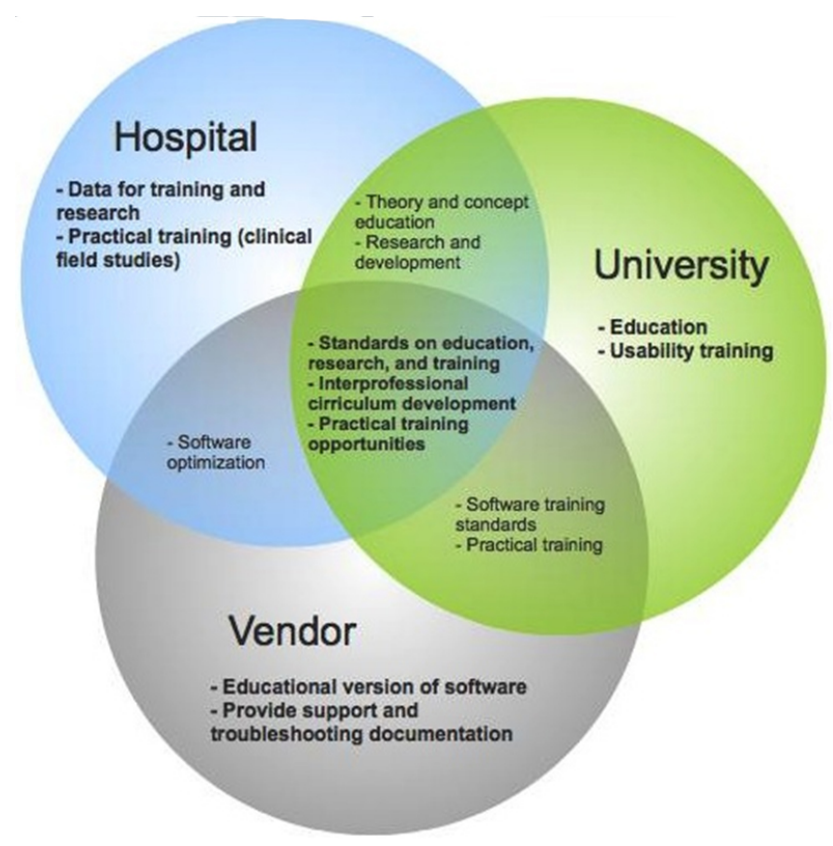

Figure 3. The roles of organizations with regards to informatics workforce training

Vendors play an essential role in training emerging informaticians by providing the necessary tools that students utilize to gain practical experience. Some major vendors provide educational and training instances of their "off the shelf" product, which focus on the basic capabilities of the software and eliminates complexities for novice users. The key element to note is that it is not necessary to expose students to all, or many, EHR systems, because due to the ONC EHR Certification Program, EHR are required to have basic functionalities deemed by the ONC HIT Standards Panel to meet the basic requirements of meaningful use and therefore quality care. Hence, training students on one certified EHR provides adequate training and prepares students to use other EHR system as well. For example, Computer Science students who learned $\mathrm{C}$ language during Freshmen year can easily pick up other programming languages, given they learn the syntax; the reason being most programming uses the same logistics and problem-solving skills.

Healthcare organizations complete the interprofessional model. Most healthcare organizations have deployed an EHR system that was built and supported by an outside vendor. It is crucial for senior leadership in healthcare organizations to realize the importance of data to train future informaticians and clinicians. An EHR system that has incomplete or synthetic data will be a barrier towards high quality education. Organizations must cooperate with institutions by providing a subset of their dataset for education, training, and research purposes, and it is critical for healthcare organizations to work with academic institutions to provide student internship opportunities to further develop informatics among the future workforce. This cooperation will benefit healthcare organizations since students are being trained on real-life scenarios, which means that healthcare organizations will spend less time and money to train new hires (see Figure 3).

When discussing informatics workforce training, two main issues must be addressed namely, EHR competency levels and interprofessional curriculum development. ${ }^{[15]}$ EHR core competencies differ from one discipline to another - each discipline's competency requirements should be considered when developing an informatics curriculum. There are general and specific core competencies for informatics. Kulikowski et al. outline specific informatics competencies, including the ability to "Understand, and analyze the types and nature of biomedical data, information, and knowledge". ${ }^{[5]}$ Depending on the type of program and the roles for which the program is educating students, these core competencies can be tailored and demonstrated in unique ways. For example, for those programs educating medical informaticians, the program may choose to educate professionals on methodologies for querying their specific clinical information systems in an effort to better understand their patient population, which in fact is a Menu Measure within the EHR Incentive Program. Other programs that are focused on educating informaticians geared towards clinical research, would most likely meet this core competency in much different way, such as analyzing extremely large datasets for predictive modeling of large outcomes. Other general core competencies may include the ability to utilize all basic functionalities of an EHR system. Again, these competencies could be tailored based upon the type of program. Therefore, a successful interprofessional curriculum should improve teamwork skills, improve awareness of other team member's roles and responsibilities, and provide training on team notes, as well as include specialty- 
specific core competencies.

The board certification in the subspecialty of clinical informatics and the advanced interprofessional informatics certification (AIIC) must be considered when discussing specific requirements for interprofessional health informatics curriculum. The American Board of Preventative Medicine is sponsoring the board certification in the subspecialty of clinical informatics. Physicians who are currently certified by the American Board of Medical Specialties (ABMS), graduated from a medical school in the US or one deemed satisfactory to the Board, has an unrestricted and currently valid license to practice medicine, and completed significant work in clinical informatics or underwent a fellowship training program in clinical informatics are eligible to sit for the board exam. ${ }^{[16]}$ The AIIC is currently under discussion by an AMIA taskforce that was assigned by the AMIA academic forum. The purpose of this certification is to "ensure that health informatics training programs adequately prepare graduates for the growing role of information and communications technology in health care organizations and the health sector at-large". [17] The AIIC is meant to be a pathway to certifica- tion for individuals that are not eligible for the subspecialty certification. The AIIC will be offered at the graduate level and based on the same core content and rigor used for the subspecialty certification. Through a partnership between AMIA and Commission on Accreditation for Health Informatics and Information Management Education (CAHIIM), specific requirements course content and graduate competencies for a graduate education program will be determined in order for graduates to sit for the certification exam.

Gardner and colleagues published a white paper in JAMIA that outlines core content for the clinical informatics subspecialty. ${ }^{[18]}$ Although the core content is likely to change as final agreements for requirements are made, it is indicated that the same core content for the clinical informatics subspecialty would also apply to the AIIC. ${ }^{[17]}$ As outlined by Gardner et al. the core contact covers four main areas: the fundamentals, clinical decision-making and care process improvement, health information systems, and leadership and management of change. ${ }^{[18]}$ Each of these areas emphasizes specific content related to clinical informatics (see Table 1).

Table 1. Core content for the Clinical Informatics Sub-specialty as outlined by Gardner et al. ${ }^{[1]}$

\begin{tabular}{ll}
\hline Core content & Topics \\
\hline Fundamentals & Clinical informatics; The Health System \\
Clinical Decision Making and Care & Clinical Decision Support; Evidence-based Patient Care; Clinical Workflow Analysis, Process \\
Process Improvement & Redesign, and Quality Improvement \\
& Information Technology Systems; Human Factors Engineering; Health Information Systems and \\
Health Information Systems & Applications; Clinical Data Standards; Information System Lifecycle \\
& Leadership Models, Processes and Practices; Effective Interdisciplinary Teams; Effective \\
& Communications; Project Management; Strategic and Financial Planning for Clinical Information \\
Leading and Managing Change & Systems; Change Management \\
\hline
\end{tabular}

\subsection{Challenges in health informatics training programs}

The largest challenge in training informatics workforce is the varying levels of knowledge and skills among trainees. Clinicians, or trainees with a clinical background, possess a strong grasp of clinical knowledge but usually lack understanding of IT tools and methods; and trainees with IT background have the ability to understand computer science concepts and utilize computational methods to solve problems, yet they tend to lack clinical knowledge such as the organization of the healthcare system, clinical workflows, terminologies, etc. For that reason the CCC program offered two distinct tracks based on a student's background: (1) clinical track, (2) IT track, shown in Figure 3. The aim of each track was to provide trainees with knowledge that trainees may not otherwise acquire and that are necessary for informaticians to be successful. While the CCC program aimed to accomplish interprofessional education by offering a clinician/practitioner consultant track and a technical/software support trainer track, the major limitation with those educational programs is the overall lack of access to vendorsupplied technology solutions and the limited exposure to interprofessional experiences through practical experiences with healthcare organizations that provide an opportunity to apply knowledge and demonstrate competencies within a semi-controlled environment.

As the board certification for the subspecialty in clinical informatics and AIIC are developed further, the distinction in curriculum requirements for clinicians vs. non-clinicians may become clearer. However, there are core requirements that must be emphasized regardless of an individual's background.

\section{Discussion}

This paper proposes a framework below that emphasizes a training program that emphasizes core requirements for the 
profession and fundamentals that may be required based on the trainees background (see Figure 4).

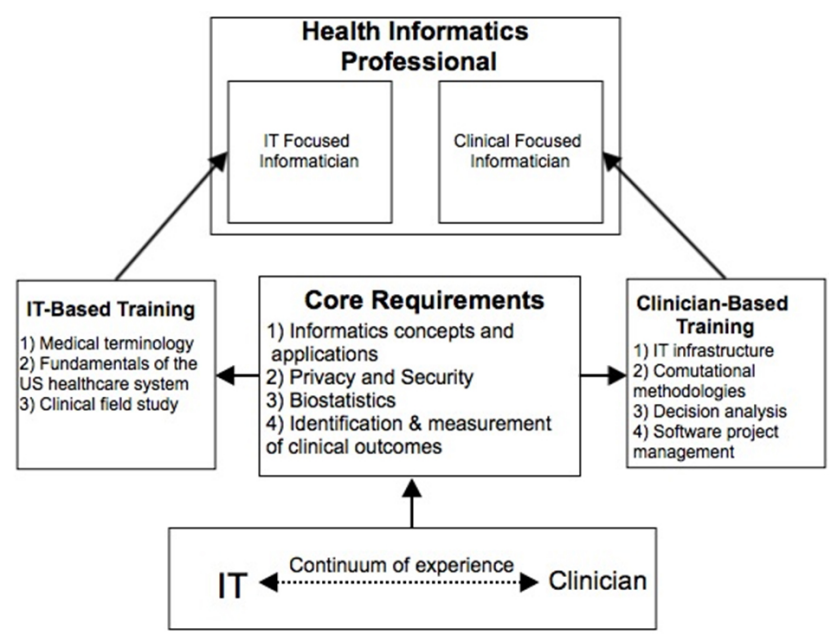

Figure 4. Informatics education and training framework

Looking at the clinical track, trainees should complement their clinical knowledge with an understanding of IT. It is essential to mention that the aim of the clinical track is not to produce programmers and developers. Rather, this track should prepare trainees to understand how to use informatics tools and resources. First, a clear understanding of health information systems will prepare clinicians to be informatics content experts. Specifically, trainees should learn about HIT such as system design and architecture, networks, security, data storage and acquisition, and HIE. Second, students should be aware of the various computational methods used in HIT systems such as decision trees, rule-based systems, database management system, data mining, and natural language processing techniques. The aim is to provide students with comprehensive understanding of the various computer science methods, as well as train them on choosing the correct set of methodologies to solve a given problem. Third, there is great need for the integration of decision analysis as part of the informatics training, the science behind decision making and analysis is the nucleus of most clinical tasks, and there needs to be more emphasis on training future informaticians about this discipline. Students should understand the philosophy, theory, methodology, and professional practice necessary to address important clinical decisions. Finally, software project management is a field that most clinicians were not exposed to prior to their informatics training and therefore, there needs to be formal training on building and managing software engineering projects. During software project management courses, small groups of trainees should work together on four main components: plan, design and develop, implement, and test. Furthermore, providing trainees with an opportunity to work with a client on a real-life project is more useful than hypothetical projects. The reason being, trainees are than exposed to actual project management challenges such as budget, time constraints, risk management, software management, as well as team communication and coordination.

With regards to the IT track, IT-based students have received extensive computer science training including design, developing, and project managing skills. However, most trainees lack a basic healthcare understanding. For that reason, IT professionals need to receive education regarding the healthcare system. The first priority is to expose trainees to fundamental concepts of the US healthcare system, trainees should understand who the providers are, how are payments made, regulations and policies, drug efficacy and safety, new healthcare reform acts, and health insurance. Second, trainees should be familiar with the various standardized terminology systems such as International Classification of Diseases (ICD), Systematized Nomenclature of Medicine - Clinical Terms (SNOMED CT), Logical Observation Identifiers Names and Codes (LOINC), RxNorm, etc. Most healthcare systems use these terminology protocols as a consistent way to index, store, retrieve, and aggregate clinical data across specialties and sites of care, and it is essential for trainees to be proficient in these terminology classifications. Finally, all IT-based trainees must conduct a clinical field study where trainees spend a substantial amount of time in a clinical setting observing, analyzing, and interacting with the clinical team. This eye-opening experience enriches the knowledge base of trainees about the structure of the clinical team, performed tasks, clinical workflow, barriers, and limitations in use of technology. The field study will bring trainees closer to the clinical world and improves collaboration and communication.

\subsection{Usability training}

Education is the foundational element towards building a strong informatician; training is the pillar that holds up an informatician. Congruent to the formal, didactic educational path, there must be a robust training environment where trainees put the theories and concepts learned into practice. Usability labs should be built to facilitate case-based scenarios where groups of trainees are formed. Each group should include trainees from different specialties and each team member should be assigned a role that is similar to their responsibilities in a real life scenario. The aim of these simulation exercises is to promote better intra-team communication and coordination, know the roles and responsibilities of other members, and understand how to effectively use technology to communicate or to conduct tasks. ${ }^{[8]}$ Cross- 
disciplinary training programs are key elements towards a successful training program. The ability to work on real case scenarios among inter-professional teams through utilizing various technologies will expedite job orientation and training periods.

The framework described above is meant to overcome some of the limitations of the current ONC training programs in preparing the workforce for informatician roles. This framework provides a bipartisan approach to informatics training by dividing the educational curriculum by specialty, rather than the current one-size fits all process. A HRSA white paper provided an analogous example within the public health informatics profession. The report stated how there is a need for distinct training programs for different roles in the public health informatics profession; one track should focus on training to a level of a Public Health informatician (worker) and another to senior Public Health informatician (supervisor). ${ }^{[19]}$ A problem with the ONC training program was the lack of content requirements for the specific roles. As stated above, the CCC and UBT had diverse curricula and offered training for roles they decided were within the scope of their curriculum. In this paper, a framework is proposed that is closely aligned with the AMIA specifications for core content for the clinical informatics sub-specialty and the AIIC.

The skillset of an informatician should encompass a set of competencies mentioned in the previous sections that prepare the professional to meet new challenges in the Health Informatics environment. The competencies, to summarize, will include familiarity with continuous quality improvement, assessment of clinical outcomes, clinical decision support, and translational data analysis for knowledge discovery in the clinical arena. An informatician may develop expertise in a specific area such as picture archiving and communication systems (PACS) or laboratory medicine but a broad-based training that engenders familiarity with the breadth of clinical health informatics will be necessary to prepare the informatician for new challenges that will arise over the course of a professional career.

\subsection{Informatics training internationally}

The proposed educational and training module applies to international workforces as well. Reports show that there is significant shortage in the informatics workforce. The English National Health Service (NHS) reports that the informatics workforce makes up 25,000 full-time employees out of 1.3 million worked in the English NHS. ${ }^{[20]}$ Similar studies reported that the health informatics workforce size in Australia is approximately 12,000 employees out of approximately 550,000 employees in health occupations, and in Canada about 32,000 HIT professionals. ${ }^{[21-23]}$ According to Hersh and Wright the US HIT workforce is comparable to those in England and Australia. ${ }^{[24]}$ However, there is a great disparity in terms of adoption of health technology in practice. According to one study, $80 \%$ of UK physicians used electronic medical records in 1996. ${ }^{[24]}$ The greatest difference between the US and other countries with high levels of HIT adoptions that have high rates of adoption of HIT within acute and ambulatory care settings is the level of financial investment in health technology. As of 2006, the US had invested approximately $\$ 43$ per capita on health technology, whereas the UK had invested \$193, Canada had invested \$32, and Germany had invested $\$ 21$ per capita. ${ }^{[24]}$ The worldwide growth of HIT highlights the need for larger numbers of well-trained professionals who understand healthcare, workflow, information and communication exchange. The problem remains in identifying the differences between HIT and Health Informatics workforces, the overlapping perception of both workforces is a challenge that will naturally dissolve with the healthcare industry reaching technological maturity. However, it is the responsibility of educators, clinicians, and vendors to promote the role of IT and Informatics in Healthcare.

In developing countries, the challenges mentioned earlier remain plus infrastructure, training, and research challenges. ${ }^{[22]}$ Attempts to adopt EHR are still modest in many nations; while EHRs are the nucleus of most Informatics work, it is a challenge to provide adequate training and education without exposure to EHRs. In the field of Health Informatics, data is the treasure; therefore, a cultural change is paramount. The adoption and utilization of technology does not suffice to solve healthcare challenges. Better collection, organization, and analysis of data while improving clinical workflow and overall efficiency and care outcomes is the ultimate goal. This can only be accomplished with a well-trained workforce that covers all sub-disciplines of health informatics.

There exist international informatics training efforts that aim to develop courses to better train informatics workforce. The Biomedical Research Informatics for Global Health Training Program (BRIGHT) is a synergy between faculty from Brazil, Mozambique, and the USA. The program started in 1999 and is still underdeveloped; the program follows a single pathway structure where all trainees receive the same courses, attend lectures and conferences, and publish papers. ${ }^{[25]}$

In 2013, the Informatics Europe and ACM Europe Working Group, which comprises scientist from various European countries, published a report on informatics Education. The report refers to Informatics as the "entire set of scientific 
concepts that make information technology possible". ${ }^{[26]}$ In the report, authors state that there is no standard informatics curriculum for the whole of Europe and therefore, authors suggest that informatics curriculum should include two principles: (1) leverage student's creativity, and (2) emphasize quality. While the concept is agreeable, it is insufficient to rely on these two principles when designing graduate level education, especially in an emerging field.

The most populous country in the world, China, has only four programs offering $\mathrm{PhD}$ degrees in Health Informatics, three programs of which lie within the same institution. ${ }^{\text {[27] }}$ A chief complaint is the absence of skilled workers who understand both health care and computing thus, there remains a need for a standard bilateral informatics curriculum that trains skilled workers. In 2002, the China Health Informatics Working Conference reported that one of the challenges is the lack of health informatics standards, as well as the lack of specialized departments of health informatics. ${ }^{[28]}$

Health Informatics in the Middle East has seen modest attempts to introduce health informatics. In 2006, the Saudi Association for Health Information (SAHI) was established to practice public activities, develop theoretical and applicable knowledge, and provide scientific and applicable studies. ${ }^{[29]}$ However, SAHI has not reported on an educational framework to be utilized by institutions in the region.

Moving on, the first step towards better healthcare is effective use of certified EHRs. International institutions are encouraged to adopt open-source EHRs; this will facilitate interface interaction as well as backend research and programming. Implementing case-based exercises and other EHR activities in the curriculum require a user-friendly interface that allows students to easily navigate through the system. Similarly, for applied courses and/or research projects students must be able to have access to the backend of the system in order to execute informatics methods such as run database queries or apply data mining algorithms. Due to the newly born status of Health Informatics, one challenge is the scarcity of well-trained faculty on the design, functionality, and operations of EHR systems. This challenge will fade away as the supply of highly trained professors increases, through the establishment of more Doctorate programs.

The new informatics training tips proposed in this paper are congruent with the basic International Medical Informatics Association (IMIA) recommendations, such as the need for healthcare professionals to acquire information storage and retrieval skills, and to receive adequate training in information and communication technology. ${ }^{[30]}$ The model proposed in the paper builds of this foundation and adds that there is a necessity for tailored training tracks that is customized to accommodate and expand professional's background, education, and training. The aim is to bridge the current gap between the supply and demand of formally-trained informaticians. This approach will substantially increase the breadth and depth of the informatics workforce, and will present to the community with well-trained professionals who will no longer require the current heavy training modules required by health organizations.

\section{Conclusions}

Current HIT workforce development programs have not established if graduated trainees have met the expectations of employers. In addition, evidence is lacking with regard to the impact of the training programs on job placement or employee status of graduated trainees. Therefore, there is not only a need to improve the evaluation methods of current HIT workforce training programs but also a need to develop a future educational framework based on the available evidence regarding workforce needs and the diversity of incoming students. Thus, an educational framework was developed that focuses on two primary tracks (IT-based and clinician-based) to fulfil the gaps in knowledge of the incoming trainees based on the prior professional experience. Also, students can be better prepared for the workforce by real work experience through clinical field studies and through evaluating competencies through usability training. Finally, our framework emphasizes the need for collaboration between EHR vendors, HCOs, and universities in order to develop appropriate curriculum integrating current HIT tools to meet the needs of the workforce. In short, informaticians need to train on current health information technologies with an emphasis on the areas in which previous exposure lacks. During training, students need to learn to assimilate and synthesize new knowledge in a wide variety of disciplines related to and including health informatics. Tailored, transdisciplinary biomedical and health informatics education is paramount to develop a workforce that can engage and contribute in an ever-evolving health care field that embraces new technologies and faces new challenges.

\section{Conflicts of Interest Disclosure}

The authors declare they have no conflicts of interest. 


\section{REFERENCES}

[1] Blumenthal D. Launching HITECH. N Engl J Med. 2010; 362(5): 382-385. http://dx.doi.org/10.1056/NEJMp0912825

[2] Furukawa MFVD, Swain M. HITECH and Health IT Jobs: Evidence from Online Job Postings. 2012 (Retrieved November, 2013). Available from: http://www.healthit.gov/sites/default/f iles/pdf/0512_0NCDataBrief2_JobPostings .pdf

[3] NORC. Implementation of ONC's workforce development program: Evaluation of the IT professionals in health care ("Workforce") program. 2012 (Retrieved March 13, 2013). Available from: http://www.healthit.gov/sites/default/files/p $\mathrm{df} /$ onc-workforce-development-program.pdf

[4] Mohan V, Abbott P, Acteson S, et al. Design and evaluation of the ONC health information technology curriculum. J Am Med Inform Assoc. 2013. http://dx.doi.org/10.1136/amiajnl-2013-0 01683

[5] Kulikowski CA, Shortliffe EH, Currie LM, et al. AMIA Board white paper: definition of biomedical informatics and specification of core competencies for graduate education in the discipline. J Am Med Inform Assoc. 2012; 19(6): 931-938. http://dx.doi.org/10.11 36/amiajnl-2012-001053

[6] Panel IECE. Core competencies for interprofessional collaborative practice: Report of an expert panel. Washington, D.C.: Interprofessional Education Collaborative; 2011.

[7] HealthIT.gov. Basics of Health IT. 2013a (Retrieved September, 2013). Available from: http://www.healthit.gov/patie nts-families/basics-health-it

[8] Friedman CP. What informatics is and isn't. J Am Med Inform Assoc. 2013; 20(2): 224-226. http://dx.doi.org/10.1136/amiajnl $-2012-001206$

[9] Otero P, Hersh W, Jai Ganesh AU. Big data: are biomedical and health informatics training programs ready? Contribution of the IMIA working group for health and medical informatics education. Yearb Med Inform. 2014; 9(1): 177-81. PMid: 25123740. http://dx.doi.org/10.15265/IY-2014-0007

[10] NORC. Workforce program evaluation: University-based training program site visit report. 2013 (Retrieved November 13, 2013) Available from: http://www.healthit.gov/sites/default/f iles/workforceevalfinal_ubt_sitevisitreport.pdf

[11] HealthIT.gov. Community college consortium to educate Health IT professionals. 2013b (Retrieved November, 2013). Available from: http://dashboard.healthit.gov/college/

[12] HealthIT.gov. Evaluation of ONC's workforce programs. 2013c (Retrieved November, 2013). Available from: http://www.healthit.gov/facas/sites/faca/files/Work force $\% 20$ Evaluation $\% 20 \mathrm{Brief}$ ing $\% 20$ f or $\% 20 \mathrm{FACA} \% 20 \mathrm{Commi}$ ttee $\% 2009 \% 2010$.pdf

[13] Initiative E. Issue brief: How the HITECH Act is helping generate jobs in health information technology. 2012 (Retrieved March, 2013). Available from: http://www.ehealthinitiative.org/reso urces/viewdownload/54-annual-surveys/210-workforce -brief-issue-brief-how-the-hitech-act-is-helping-g enerate-jobs-in-health-information-technology.html

[14] Patel VL, Yoskowitz NA, Arocha JF, et al. Cognitive and learning sciences in biomedical and health instructional design: A review with lessons for biomedical informatics education. [Research Support, Non-U.S. Gov't Review]. J Biomed Inform. 2009; 42(1): 176-197. http://dx.doi.org/10.1016/j.jbi.2008.12.002

[15] Hersh W. The health information technology workforce: estimations of demands and a framework for requirements. Appl Clin Inform. 2010; 1(2): 197-212. http://dx.doi.org/10.4338/ACI $-2009-11-\mathrm{R}-0011$

[16] AMIA. American Board of Preventive Medicine Clinical Informatics Subspecialty Board Exam. 2013b (Retrieved November, 2013). Available from: http://www.amia.org/clinical-informati cs-board-review-course/board-exam

[17] AMIA. Advanced Interprofessional Informatics Certification Task Force. 2013a (Retrieved November, 2013). Available from: http://www.amia.org/programs/academic-forum/e xecutive-committee/task-force

[18] Gardner RM, Overhage JM, Steen EB, et al. Core content for the subspecialty of clinical informatics. J Am Med Inform Assoc. 2009; 16(2): 153-157. http://dx.doi.org/10.1197/jamia.M3045

[19] HRSA. Competencies for public health informatics. 2013 (Retrieved March, 2013). Available from: http://www.cdc.gov/Informat icsCompetencies/downloads/PHI_Competencies.pdf

[20] Eardley T. NHS Informatics Workforce Survey. ASSIST: London, England; 2006.

[21] AIHW. Australia's health. 2010 (Retrieved September, 2013). Available from: http://www . aihw.gov.au/health-workforce/

[22] Hersh W, Margolis A, Quiros F, et al. Building a health informatics workforce in developing countries. [Research Support, N.I.H., Extramural]. Health Aff (Millwood). 2010; 29(2): 274-277. http: //dx.doi.org/10.1377/hlthaff.2009.0883

[23] O'Grady J. Health informatics and health information management: Human resources report. Toronto, Ontario: Prism Economics and Analysis; 2009.

[24] Hersh W, Wright A. What workforce is needed to implement the health information technology agenda? Analysis from the HIMSS analytics database. AMIA Annu Symp Proc. 2008; 303-307. PMid: 18998990.

[25] Brasil, U. A. d. BRIGHT: Biomedical Research Informatics for Global Health Training Program. 2013 (Retrieved November, 2013). Available from: http://www.ohnomachado.net/program.html

[26] Group IEAEW. Informatics education: Europe cannot afford to miss the boat. Germany Chapter of the ACM. 2013.

[27] Hsu HM, Hou YH, Chang IC, et al. Factors influencing computer literacy of Taiwan and South Korea nurses. [Validation Studies]. J Med Syst. 2009; 33(2): 133-139. PMid: 19397098. http: //dx.doi.org/10.1007/s10916-008-9173-5

[28] Chen XH. Paper 2 in China Informatics Working Conference. PRC Ministry of Health. 2002.

[29] SAHI. Saudi Association for Health Informatics. 2011 (Retrieved November, 2013). Available from: http://www.sahi.org.sa

[30] Mantas J, Ammenwerth E, Demiris G, et al. Recommendations of the International Medical Informatics Association (IMIA) on Education in Biomedical and Health Informatics. First Revision. Methods Inf Med. 2010; 49(2): 105-120. http://dx.doi.org/10.3414/ME5 119 